\title{
Wireless Remote Monitoring of Packaged Passive Sensor for In-situ Pres- sure Measurement in Highly Reflective Environments
}

\author{
J. Philippe, D. Henry, M. V. De Paolis, A. Rumeau, A. Coustou, S. Charlot, P. Pons and H. Aubert \\ LAAS-CNRS; University of Toulouse; CNRS, INP \\ 7, Avenue Roche, Toulouse, France
}

\begin{abstract}
This communication reports the design of a packaged passive microwave sensor for measuring applied pressures up to 3bars in highly reflective (metallic) environments. The technological fabrication process is detailed as well as the packaging of the device, which shows an outstanding 440MHz/bar sensitivity between 0.75 and 2.8bars. A microwave Frequency Modulated Continuous Wave (FM-CW) radar is then used for remotely reading the applied pressures. A large full-scale range of $12 \mathrm{~dB}$ is obtained with a sensitivity of $5.7 \mathrm{~dB} / \mathrm{bar}$ and an estimated precision of $\pm 10 \mathrm{mbars}$ between 0.5 and 2 bars.

Index Terms - wireless sensors, pressure sensors, FM-CW radar reader, highly reflective environments.
\end{abstract}

\section{INTRODUCTION}

Numerous developments are reported in the literature on passive and wireless sensors for measuring various physical quantities, such as temperature, humidity, stress, gas, and pressure [1-5]. Many proof-of-concepts have been demonstrated using not-packaged sensing device and non-portable measurement set-up, such as probe station and Vector Network Analyzer (VNA). Only few papers have been devoted to the packaging of passive microwave sensors as well as the wireless interrogation of these sensors in highly reflective and/or industrial environments. The device designed here allows portable detection set-up for the in-situ pressure measurements in such environments. This communication will first detail the pressure sensor operating principle. Both its technological fabrication process and packaging are addressed. The fabricated sensor is then characterized and remotely interrogated using a microwave FM-CW radar. The use of this sensor in the practical context of high reflective environments is finally discussed.

\section{Passive Microwave Pressure Sensors : Design AND MEASUREMENT RESULTS}

The working principle of this pressure sensor is reported in [2]. A planar resonator is located inside a cavity and below a flexible diaphragm. The applied pressure creates a deflection of the membrane, modifies the gap between the membrane and the RF structure, and consequently changes the resonant frequency of the resonator. The physical quantity can be in principle derived from the measurement of this frequency. The dimensions of microstrip resonator, cavity and membrane are determined for addressing applied pressure in a range up to 3bars.

The microwave transducer is fabricated using microelectronic technology. Both structure and dimensions of the transducer are given in Fig. 1 and Fig. 2. A $0.05 \mu$ m thick titanium
(Ti) and a $0.5 \mu \mathrm{m}$ thick copper $(\mathrm{Cu})$ layers are first deposited on a $500 \mu \mathrm{m}$ thick borosilicate glass (B33) substrate to fabricate a half-wavelength microstrip resonator. An additional step of $\mathrm{Cu}$ electrochemical growth increases the resonator ports thickness until $9 \mu \mathrm{m}$. A final thin gold ( $\mathrm{Au}$ ) layer deposition by electroless technique allows the $\mathrm{Cu}$ structure protection against oxidation due to further high-temperature processes. The resonator is located inside a $5.5 \mathrm{~mm}$ diameter cylindrical cavity formed by a $10 \mu \mathrm{m}$ thick spincoated low-losses RF photoresist. A $100 \mu \mathrm{m}$ thick high resistivity silicon (Si-HR) membrane is then bonded on the photoresist at $100^{\circ} \mathrm{C}$. The ground plane at the bottom face is composed of a $0.05 \mu \mathrm{m}$ thick layer of chromium (Cr) and of a $1.5 \mu \mathrm{m}$ thick layer of aluminum (Al). This technological process offers a fast fabrication and provides a sufficient hermeticity necessary for demonstrating the proof-of-concept. Furthermore, it affords an excellent electrical contact with external connectors because of the $\mathrm{Cu}$ ports electrochemical growth. Both relative permittivity $\varepsilon_{R}$ and loss tangent $\tan \delta$ of the B33 substrate and the photoresist are given in Table I.

After the fabrication process, the microwave transducer is placed inside a $11.25 \times 8.3 \times 5.56 \mathrm{~mm}^{3} \mathrm{Al}$ metallic cavity (Fig. 3). The cavity is designed for not altering the device's performance. Some additional $80 \mu \mathrm{m}$ thick of brass films are placed below the transducer.

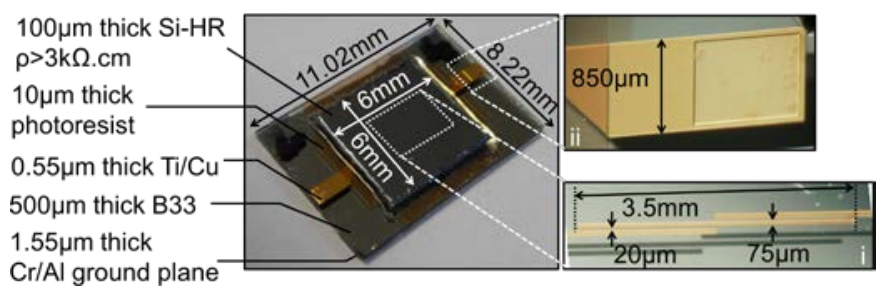

Fig. 1. Photo of the microwave sensor after bonding with its Si-HR membrane. $\rho$ denotes the resistivity parameter. The insets (i) and (ii) show the microstrip half-wavelength resonator below the membrane and the input or output port $(9 \mu \mathrm{m}$ thick $\mathrm{Cu})$ of the resonator, respectively.

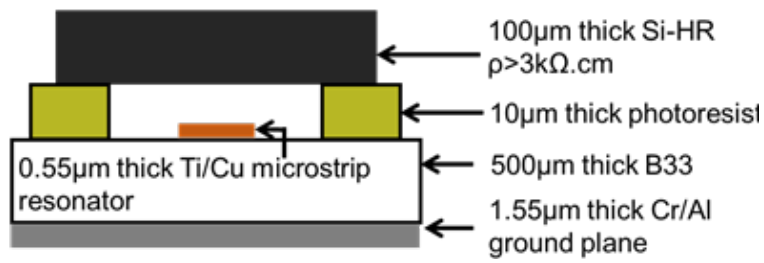

Fig. 2. Cross-sectional view of the different sensor layers. $\rho$ denotes the resistivity parameter. Both relative permittivity $\left(\varepsilon_{R}\right)$ and the loss tangent (tan $\delta$ ) of each material are given in Table I. 


\begin{tabular}{|c|c|c|}
\hline \multicolumn{3}{|c|}{ TABLE I } \\
\hline RF PARAMETERS OF SENSOR MATERIALS \\
\hline Material & Dielectric permittivity $\boldsymbol{\varepsilon}_{\boldsymbol{R}}$ & Loss tangent tan $\boldsymbol{\delta}$ \\
\hline B33 & 4.62 & $90.10^{-4}$ \\
\hline Photoresist & 4.60 & $37.10^{-4}$ \\
\hline
\end{tabular}
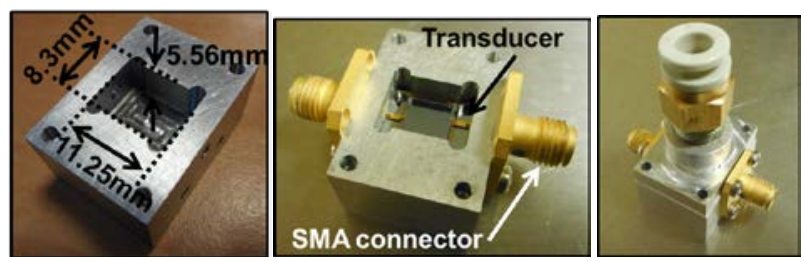

Fig. 3. Presentation of the packaging. With its package, the sensor's dimensions are of $3.6 \times 2.4 \times 4.2 \mathrm{~cm}^{3}$.

This solution allows a reliable connection between the package (metallic cavity) and transducer's ground plane, and also a convenient set-up for testing the fabricated microstrip sensors. The final dimensions of the packaged pressure sensor are of 3.6 $\mathrm{x} 2.4 \times 4.2 \mathrm{~cm}^{3}$.

Scattering parameters were first characterized at room temperature with a VNA for various applied pressures up to 3bars in order to estimate the sensitivity of the packaged transducer. Before each measurement, the pressure is first applied for $2 \mathrm{~min}$. The precision of the pressure monitoring system is $\pm 50 \mathrm{mbars}$. Fig. 4 reports the variation of the resonant frequency $f_{\text {res }}$ and of the input reflection coefficient $S_{i i}$ at a frequency $f_{c}$ of $23.8 \mathrm{GHz}$ (radar operating frequency, see Section III) as a function of the overpressure. The obtained full-scale measurement range is of $16.2 \mathrm{~dB}$ between 0 and 3bars, with a convenient linear behavior between 1.2 and 2.4bars. The measurement sensitivity is $3.4 \mathrm{~dB} / \mathrm{bar}$ in this range while it is of $440 \mathrm{MHz} /$ bar sensitivity between 0.75 and $2.8 \mathrm{bars}$ for the resonant frequency with a coefficient of determination $\mathrm{R}^{2}$ of 0.99 and 1.0 respectively. To the knowledge of the authors, this is the highest sensitivity for a wireless and passive sensor assigned for the same pressure range (Table II).

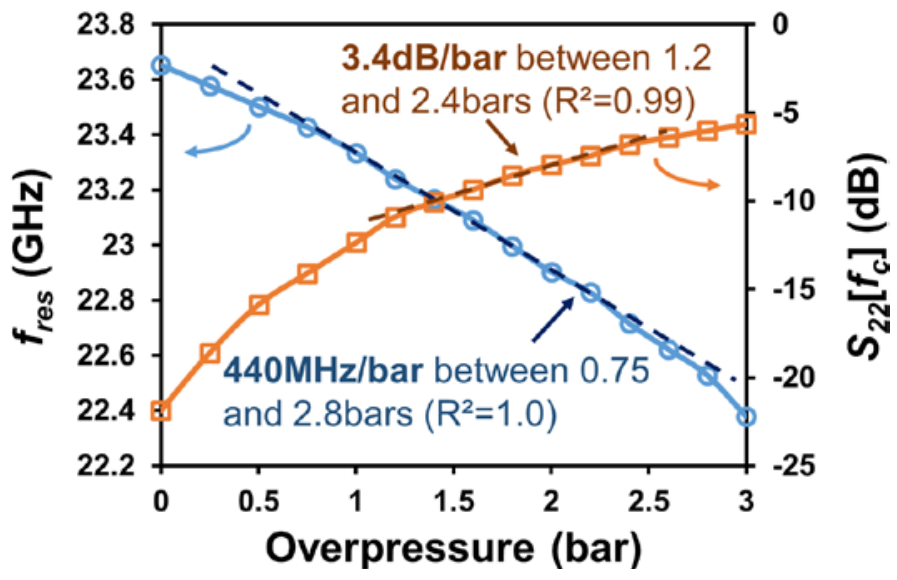

Fig. 4. Variation of the measured resonant frequency $f_{\text {res }}$ and of the measured input reflection coefficient $S_{i i}$ at $f_{c}$ of $23.8 \mathrm{GHz}$ (radar operating frequency) as a function of the overpressure.
TABLE II

COMPARISON WITH WIRELESS PASSIVE PRESSURE SENSORS

\begin{tabular}{|c|c|c|c|}
\hline Reference & Range (bar) & $\boldsymbol{f}_{\text {res }}(\mathbf{G H z})$ & Sensitivity (MHz/bar) \\
\hline$[5]$ & $0-3$ & 0.022 & 0.0256 \\
\hline$[4]$ & $0-7$ & 0.017 & 0.141 \\
\hline$[3]$ & $0.7-2$ & 0.035 & 0.625 \\
\hline$[2]$ & $0-3$ & 39 & 370 \\
\hline This work & $0-3$ & 23.6 & 440 \\
\hline
\end{tabular}

This outstanding measured sensitivity makes this sensor interesting for remote interrogation.

\section{REMOTE INTERROgATION OF THE PASSIVE PRESSURE SEN- SORS}

The principle of the remote interrogation is based on the measurement of a pressure-dependent radar echo using a FMCW radar. For this purpose, the packaged passive pressure transducer is: (a) terminated by a $50 \Omega$ load on the first port and (b) connected to a horn antenna on the second port through a delay line of effective length $L$ of $1.2 \mathrm{~m}$. As it will be shown here, the delay line is convenient to detect both the sensing mode and the sensor structural mode [6] in high reflective environments. In order to characterize the radar echo level as a function of the applied pressure, the passive sensor is placed in an anechoic chamber at a distance of $3.7 \mathrm{~m}$ from a $24 \mathrm{GHz}$ FMCW radar (IMST DK-sR-1030e model), as depicted on Fig 5.

The FM-CW radar transmits a chirp with a carrier frequency $f_{c}$ of $23.8 \mathrm{GHz}$ and a modulation bandwidth $B$ of $2 \mathrm{GHz}( \pm 1 \mathrm{GHz}$ around $f_{c}$ ) through a horn lens antenna (the resulting depth resolution $d$ is of $c /(2 B)=7.5 \mathrm{~cm}$, with $c$ the velocity of light). This antenna has a gain of $28 \mathrm{dBi}$ and a $3 \mathrm{~dB}$ beamwidth of $6^{\circ}$. The reflected signal is received by a horn antenna with a gain of $20 \mathrm{dBi}$ and a $3 \mathrm{~dB}$ beamwidth of $15^{\circ}$. The input power is of $20 \mathrm{dBm}$. The echo level of the sensing mode depends mainly on both the distance between the radar and the pressure sensor and the reflection coefficient at the input of the latter at the carrier frequency $f_{c}$. The echo level is derived from performing a Fast Fourier Transform (FFT) on the difference between the transmitted and received FM signals. The low-frequency spectrum of the signal resulting from this FFT is the so-called beat frequency spectrum. A three-dimensional (3D) mechanical beamscanning of the Tx antenna is performed $\left(-10^{\circ}\right.$ to $10^{\circ}$ with a step of $1^{\circ}$ in the azimuthal and elevation directions respectively denoted $\theta$ and $\varphi$ in Fig. 6). The beamscanning is controlled and automated by a pan-tilt connected to a computer unit. It leads to the record of 441 beat frequency spectra (one spectrum per direction). One example of such beat frequency spectrum is sketched in Fig. 6. The structural and sensing mode of the sensor are respectively detected at $3.9 \mathrm{~m}$ and $4.9 \mathrm{~m}$ from the radar. The 3D beamscanning is performed for overpressures varying from 0 to 3bars. The precision of the pressure monitoring system is also \pm 50 mbars. In Fig. 7 , the $3 \mathrm{D}$ radar echo at $4.9 \mathrm{~m}$ is displayed with isosurfaces [7] for two values of the overpressure - that are 0bar (Fig. 7(a)) and 3bars (Fig. 7(b)) - between 


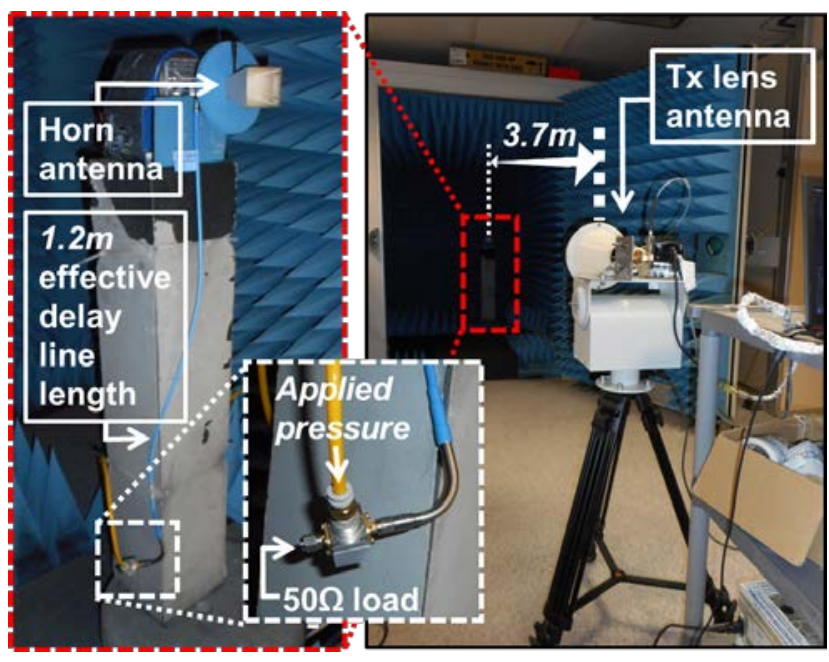

Fig. 5. Experimental set up for radar measurement.

4.5 and $5.5 \mathrm{~m}$. The maximal echo level value $e_{\text {Max }}$ detected inside this volume defines a statistical estimator from which the pressure applied on the passive transducer can be derived. As a matter of fact, the value of $e_{\operatorname{Max}}$ gradually increases with the overpressure and a dynamic range of $12 \mathrm{~dB}$ is reached between 0 and 3bars. The highest dynamic range at this distance is of $18 \mathrm{~dB}$ and is obtained with a delay line terminated by loads of $0 \Omega$ (highest echo level) and $50 \Omega$ (lowest echo level). Consequently, the passive pressure sensor has a dynamic range which is $66 \%$ lower than the highest achievable dynamic range. The estimator $e_{\text {Max }}$ is given in Fig. 8 as a function of overpressures from 0 to 3bars. A linear behavior is observed between 0.5 and 2.0bars with a coefficient of determination $R^{2}$ of 0.98 and a sensitivity of $5.7 \mathrm{~dB} / \mathrm{bar}$. The precision of this estimator, that is, the standard deviation of $e_{\text {Max }}$ for a radar echo at a given range, is computed from 50 consecutive 3D radar beamscannings. As a result, the pressure is obtained with a precision of \pm 10 mbars between 0.5 and 2.0bars.
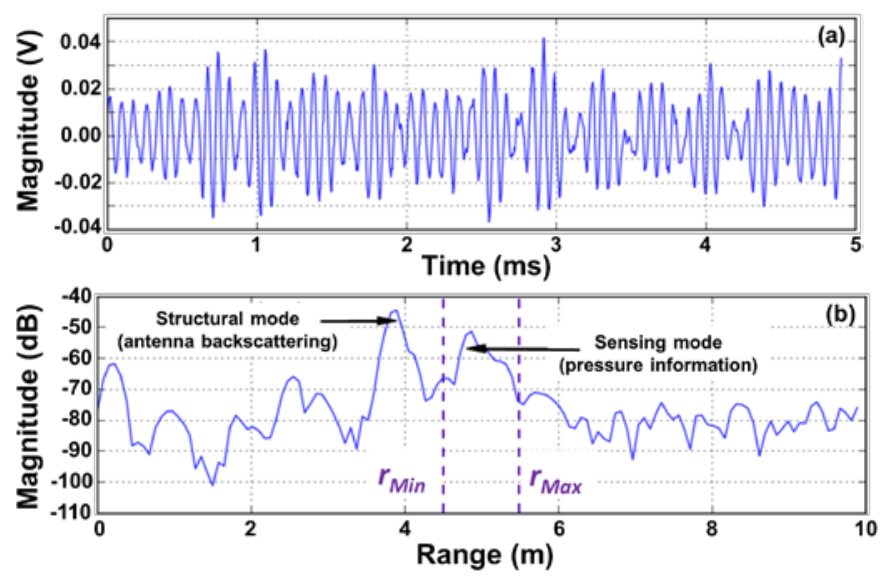

Fig. 6. (a) Time-difference signal and (b) beat frequency spectrum at a given direction of interrogation $\theta=0^{\circ}$ and $\varphi=0^{\circ}$. Structural and sensing modes are characterized by two peaks separated by a distance corresponding to the effective length of the delay line. To retrieve the pressure, we focus on a range window between $4.5 \mathrm{~m}$ and $5.5 \mathrm{~m}$ noted $r_{\text {Min }}$ and $r_{\text {Max }}$, respectively.

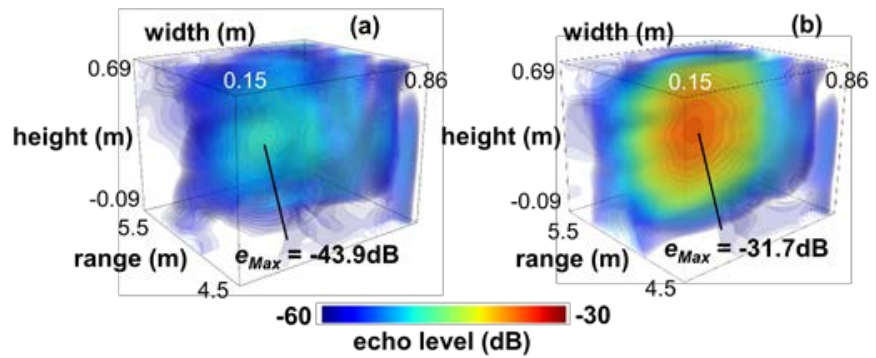

Fig 7. 3D echo level of the passive pressure sensor displayed with isosurfaces (echo level with same values) for an overpressure of Obar (a) and 3bars (b). The statistical estimator $e_{\text {Max }}$ of the pressure is defined here as the maximal echo level detected inside the scanned volume.

The passive pressure sensor is intended to be operational in high reflective environments. In such environments, numerous clutters (parasitic echoes) may mask the radar echo of the passive transducer. To overcome this issue, the length of the delay line must be chosen to shift the radar echo of the sensing mode in a region offering a high signal-to-noise ratio (SNR).

Preliminary tests with antennas and delay lines terminated with $0 \Omega$ and $50 \Omega$ at a range of $5.0 \mathrm{~m}$ have been performed in highly reflective environment (as shown in Fig. 9).

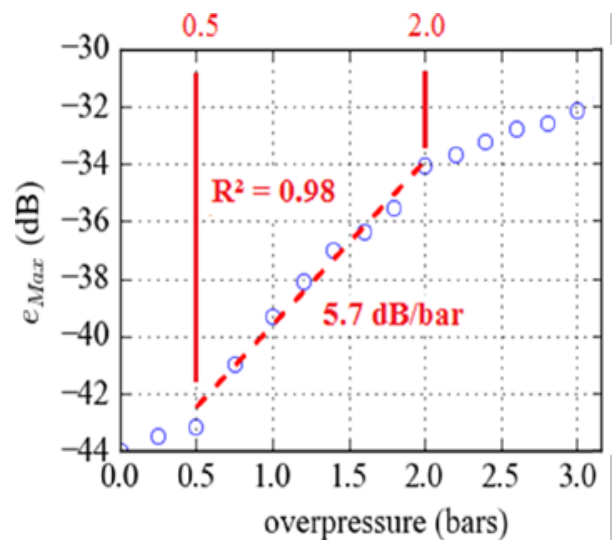

Fig. 8. Estimator $e_{\operatorname{Max}}$ (maximal echo level detected inside the scanned volume) as a function of the pressure applied on the passive pressure transducer. Between 0.5 and 2bars, a convenient linear variation is observed with a sensitivity of $5.7 \mathrm{~dB} / \mathrm{bar}$ and a coefficient of determination $\mathrm{R}^{2}$ of 0.98 . The precision is estimated at $1.3 \%$ for $e_{\text {Max }}=-42 \mathrm{~dB}$.

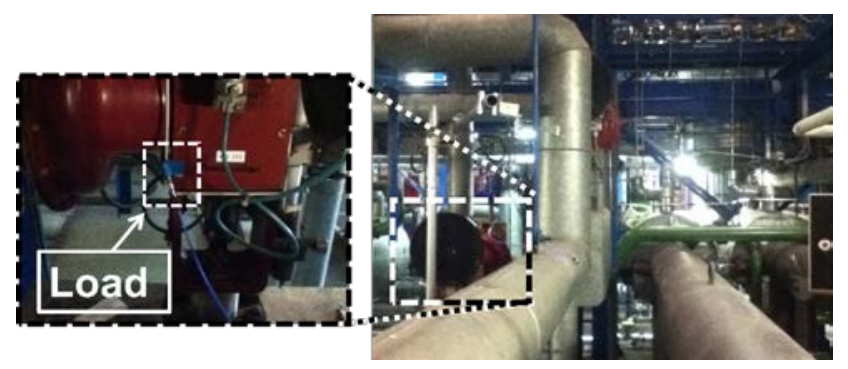

Fig. 9. Highly reflective environment used for radar detection of the sensing scattering mode. 
From these preliminary measurements, it can be derived that the radar detection and remote reading of passive pressure sensors are possible even for echo level down to -39dB. This corresponds to a minimal measurable pressure of $1.0 \mathrm{bar}$ in such highly reflective environment. These preliminary results must be completed by extensive radar experiments in such high reflective environment, but they are very encouraging and pave a way for the remote reading of passive pressure sensors in this kind of environment.

\section{CONCLUSION}

This paper reported a packaged passive and wireless pressure sensor designed for in-situ pressure monitoring in highly reflective environment. The technological process allows the fabrication of a high sensitive ( $5.7 \mathrm{~dB} / \mathrm{bar})$ pressure transducer. The $3 \mathrm{D}$ radar beamscanning technique allows the remote reading of the fabricated sensor with a measurement precision of \pm 10 mbars (1.3\%) between 0.5 and 2.0bars at the distance of 5 meters. Finally, preliminary results in a highly reflective environment are reported and pave the way of the accurate measurement of pressure in multi-path propagation environments by using fully passive and portable sensors.

\section{ACKNOWLEDGMENT}

The authors wish to thank Occitanie Region (CARANUC Project) and EDF (Electricité de France) for financial support. This work was partly supported by LAAS-CNRS micro and nanotechnologies platform members of the French RENATECH network.

\section{REFERENCES}

[1] S. Dey, J. K. Saha and N. C. Karmakar, "Smart Sensing: Chipless RFID Solutions for the Internet of Everything," in IEEE Microw. Mag., vol. 16, no. 10, pp. 26-39, Nov. 2015.

[2] M. M. Jatlaoui, F. Chebila, P. Pons and H. Aubert, "Wireless Interrogation Techniques for a Passive Pressure Micro-sensor using an EM transducer”, 2009 European Microwave Conference (EuMC), Rome, 2009, pp. 053-056.

[3] Q. Tan, T. Luo, T. Wei, J. Liu, L. Lin and J. Xiong, “A Wireless Passive Pressure and Temperature Sensor via Dual LC Resonant Circuit in Harsh Environments”, in J. Microelectromech. Syst., vol. 26, no. 2, pp. 351-356, April 2017.

[4] M. A. Fonseca, J. M. English, M. von Arx and M. G. Allen, "Wireless micromachined ceramic pressure sensor for high-temperature applications," in J. Microelectromech. Syst., vol. 11, no. 4, pp. 337-343, Aug 2002.

[5] G. J. Radosavljevic, L. D. Zivanov, W. Smetana, A. M. Maric, M. Unger and L. F. Nad, "A wireless embedded resonant pressure sensor fabricated in the standard LTCC technology," in IEEE Sensors J., vol. 9, no. 12, pp. 1956-1962, Dec. 2009.

[6] R. F. Harrington, "Theory of loaded scatterers," in "Proceedings of the Institution of Electrical Engineers, vol. 111, no. 4, pp. 617-623, April 1964.

[7] P. Ramachandran and G. Varoquaux, "Mayavi: 3D Visualization of Scientific Data," in Computing in Science \& Engineering, vol. 13, no. 2, pp. 40-51, March-April 2011. 